\title{
Comparing minimal simplicial models
}

\author{
Michał Adamaszek
}

Received: 1 January 2012 / Accepted: 18 July 2012 / Published online: 8 August 2012

(C) Tbilisi Centre for Mathematical Sciences 2012

\begin{abstract}
We compare minimal combinatorial models of homotopy types: arbitrary simplicial complexes, flag complexes and order complexes. Flag complexes are the simplicial complexes which do not have the boundary of a simplex of dimension greater than one as an induced subcomplex. Order complexes are classifying spaces of posets and they correspond to models in the category of finite $T_{0}$-spaces. In particular, we prove that stably, that is after a suitably large suspension, the optimal flag complex representing a homotopy type is approximately twice as big as the optimal simplicial complex with that property (in terms of the number of vertices). We also investigate some related questions.
\end{abstract}

Keywords Triangulation $\cdot$ Simplicial complex $\cdot$ Minimal model $\cdot$ Homotopy type

Mathematics Subject Classification (1991) $\quad 55 \mathrm{P} 10 \cdot 55 \mathrm{U} 10 \cdot 55 \mathrm{P} 40$

\section{Introduction}

Whenever we have a combinatorial category which models topological spaces we can ask about the minimal size of models. In this short note we study the following numbers defined for a topological space $X$ :

Communicated by Graham Ellis.

Research supported by the Centre for Discrete Mathematics and its Applications (DIMAP), EPSRC award EP/D063191/1.

M. Adamaszek $(\varangle)$

Mathematics Institute and DIMAP, University of Warwick, Coventry CV4 7AL, UK

e-mail: aszek@mimuw.edu.pl 


$$
\begin{aligned}
& m_{s}(X)=\min \{\# V(K): K \text { is a simplicial complex with } K \simeq X\}, \\
& m_{f}(X)=\min \{\# V(K): K \text { is a flag simplicial complex with } K \simeq X\}, \\
& m_{p}(X)=\min \{\# P: P \text { is a poset with } \Delta(P) \simeq X\} .
\end{aligned}
$$

The symbol $\simeq$ denotes homotopy equivalence. We do not distinguish between an abstract simplicial complex and its geometric realization. The notation $\Delta(P)$ stands for the order complex of a poset $P$, that is the simplicial complex whose faces are the chains of $P$. Its geometric realization is the classifying space $\mathcal{B P}$ of $P$. By $\# V(K)$ (resp. $\# P$ ) we denote the number of vertices in $K$ (resp. the number of elements in $P$ ). A simplicial complex is flag if its every minimal non-face is of dimension 1 or, equivalently, if it is the maximal simplicial complex with the given 1-skeleton. Moreover, by the results of McCord and Stong (see $[12,10]) m_{p}(X)$ is equal to the minimal number of points in a finite $T_{0}$-space weakly equivalent to $X$. We say a space $X$ is of finite type if $m_{s}(X)<\infty$. Computing the values of $m_{s}(X)$ and $m_{f}(X)$ is related to the rather classical problem of finding minimal triangulations of spaces (here up to homotopy). The properties of $m_{p}(X)$ were studied in [3] through the perspective of finite $T_{0}$-spaces. Note that by definition $m_{s}(X), m_{f}(X)$ and $m_{p}(X)$ are invariants of the homotopy type of $X$.

We have the following obvious inequalities

$$
m_{s}(X) \leq m_{f}(X) \leq m_{p}(X) \leq 2^{m_{s}(X)} .
$$

The second one follows since the order complex of a poset is always flag, and the third one is a consequence of the fact that every simplicial complex is homeomorphic to the order complex of its own face poset. One motivation for this work is to see how far $m_{f}(X)$ can exceed $m_{s}(X)$.

Define the homological dimension of $X$ as

$$
h(X)=\max \left\{k: \widetilde{H}_{k}(X ; \Lambda) \neq 0 \text { for some group } \Lambda\right\}
$$

where $\widetilde{H}_{k}(X ; \Lambda)$ denotes the reduced homology groups with coefficients in $\Lambda$. If $X$ is acyclic, i.e. all its reduced homology groups vanish, we leave $h(X)$ undefined. Then we have the following proposition.

Proposition 1.1 For any non-empty, non-acyclic space $X$ we have

$$
m_{s}(X) \geq h(X)+2, \quad m_{f}(X) \geq 2 h(X)+2, \quad m_{p}(X) \geq 2 h(X)+2 .
$$

Moreover, in each of those inequalities equality holds if and only if $X \simeq S^{n}$ for some $n \geq 0$.

The statement about $m_{s}(X)$ is obvious, the one about $m_{f}(X)$ can be found in [6] and the weaker inequality for $m_{p}(X)$ follows independently from the results of [3]. For completeness we will provide a short proof in the next section.

The last statement implies, in particular, that $\lim _{k \rightarrow \infty} m_{f}\left(S^{k}\right) / m_{S}\left(S^{k}\right)=2$. We will prove that an analogous result holds for suspensions of any space of finite type. 
Theorem 1.2 For any non-acyclic space $X$ of finite type

$$
\lim _{k \rightarrow \infty} \frac{m_{s}\left(\Sigma^{k} X\right)}{k}=1, \quad \lim _{k \rightarrow \infty} \frac{m_{f}\left(\Sigma^{k} X\right)}{k}=\lim _{k \rightarrow \infty} \frac{m_{p}\left(\Sigma^{k} X\right)}{k}=2 .
$$

Therefore

$$
\lim _{k \rightarrow \infty} \frac{m_{f}\left(\Sigma^{k} X\right)}{m_{s}\left(\Sigma^{k} X\right)}=2
$$

A heuristic counting argument suggests that there should exist spaces $X$ for which $m_{f}(X)$ is close to $2^{m_{s}(X)}$, although it is a tantalizing problem to prove this claim or find such examples. However, it is easier to show explicit examples where the values of $m_{f}(X)$ and $m_{s}(X)$ differ by more than a factor of 2 .

Proposition 1.3 For every $\epsilon>0$ there is a space $X$ with

$$
\frac{m_{f}(X)}{m_{s}(X)}>2.5-\epsilon
$$

Proposition 1.1 prompts the definition of three non-negative homotopical invariants

$$
\begin{gathered}
c_{s}(X)=m_{s}(X)-h(X)-2, \\
c_{f}(X)=m_{f}(X)-2 h(X)-2, \\
c_{p}(X)=m_{p}(X)-2 h(X)-2
\end{gathered}
$$

which are some simple measure of "complexity" of a non-acyclic space $X$. Proposition 1.1 also implies that $c(X)=0$ if and only if $X \simeq S^{n}$, where $c(X)$ is any of $c_{s}(X), c_{f}(X)$ or $c_{p}(X)$. Our last result deals with the next case, namely $c(X)=1$.

Proposition 1.4 We have the following characterization:

(a) If $L$ is any non-acyclic simplicial complex with at most $n+2$ vertices then $c_{S}\left(S^{n} \vee\right.$ $\Sigma L)=1$.

(b) $c_{f}(X)=1$ (and $c_{p}(X)=1$ ) if and only if $X \simeq S^{n} \vee S^{m}$ for some $n, m \geq 0$.

Already here we see that $c_{f}(X)$ is a finer invariant than $c_{S}(X)$.

Let us remark that the next case $c_{S}(X)=2$ is also quite interesting. The real projective space $\mathbf{P}^{2}$ satisfies

$$
m_{s}\left(\mathbf{P}^{2}\right)=6, \quad m_{f}\left(\mathbf{P}^{2}\right)=11, \quad m_{p}\left(\mathbf{P}^{2}\right) \leq 13 .
$$

The first quantity is classical and the well-known triangulation of $\mathbf{P}^{2}$ with 6 vertices is shown in Fig. 1a). The minimal flag triangulation has 11 vertices and is shown in Fig. 1b). In fact it is the smallest flag complex with torsion in homology, which was checked in [7]. It is believed that $m_{p}\left(\mathbf{P}^{2}\right)=13$ and the relevant order complex 
Fig. 1 The models of $\mathbf{P}^{2}$ as a minimal simplicial complex, minimal flag complex and minimal known order complex. The antipodal vertices (and hence the corresponding edges) on each boundary should be identified

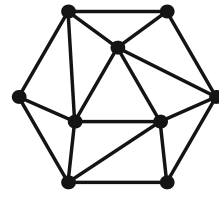

(a)

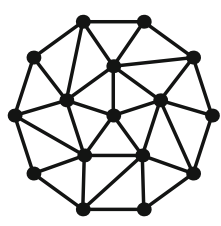

(b)

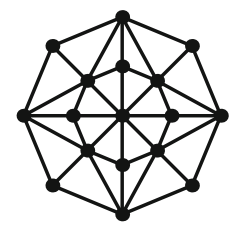

(c)

with 13 vertices is shown in Fig. 1c). ${ }^{1}$ For the corresponding poset see [4, Sect. 4], [13, Conj. 5.4] or [2, Ex. 7.1.1]. As a consequence, since $h\left(\mathbf{P}^{2}\right)=2$, we have

$$
c_{s}\left(\mathbf{P}^{2}\right)=2, \quad c_{f}\left(\mathbf{P}^{2}\right)=5, \quad c_{p}\left(\mathbf{P}^{2}\right) \leq 7 .
$$

Let us remark that one could ask for a generalization of $m_{p}(X)$ counting the minimal number of objects in any category $\mathcal{C}$ (not necessarily a poset) whose classifying space $\mathcal{B C}$ is homotopy equivalent to $X$. However, by [11], every space of finite type is homotopy equivalent to the classifying space $\mathcal{B} M$ of a discrete monoid, hence of a category with just one object.

\section{Proofs}

We start with some preliminary definitions. Let $\Delta_{n}$ be the $n$-dimensional simplex (with $n+1$ vertices) and let $\partial \Delta_{n}$ be its boundary. Then $S^{0}=\partial \Delta_{1}$ is the 0 -sphere and we define $O_{n}=\underbrace{S^{0} * \cdots * S^{0}}_{n}$ where $*$ denotes the simplicial join. The flag simplicial complex $O_{n}$ (known as the boundary of the cross-polytope) has $2 n$ vertices and it is homeomorphic to $S^{n-1}$.

The cone $C K$ over $K$ is the join $K *$ pt with a single point (the apex of the cone) and the (unreduced) suspension is $\Sigma K=K * S^{0}$. The cone $C K$ is contractible and $\widetilde{H}_{i}(\Sigma K)=\widetilde{H}_{i-1}(K)$.

If $G$ is a graph then the clique complex $\mathrm{Cl}(G)$ of $G$ is the simplicial complex whose faces are all the cliques in $G$, i.e. subsets $W \subseteq V(G)$ such that the induced graph $G[W]$ is complete. The independence complex $\operatorname{Ind}(G)$ is defined similarly, except that its faces are given by the independent sets of $G$, i.e. subsets $W \subseteq V(G)$ for which $G[W]$ has no edges. The complement $\bar{G}$ of $G$ is the graph whose edges are the non-edges of $G$. One sees that $\mathrm{Cl}(G)=\operatorname{Ind}(\bar{G})$ and that a simplicial complex $K$ is flag if and only if $K=\mathrm{Cl}\left(K^{(1)}\right)$, where $K^{(1)}$ is the 1-skeleton of $K$. For more details about these constructions consult [9]. The graphs $P_{n}, C_{n}$ will be, respectively, the path and the cycle with $n$ vertices. If $x \in V(G)$ then $N[x]$ denotes the set consisting of $x$ together with all its neighbours in $G$.

If $v$ is a vertex of any simplicial complex $K$ then we define the link $\operatorname{lk}_{K}(v)$, star $\operatorname{st}_{K}(v)$ and deletion $K \backslash v$ in the usual way [9]. Note that if $K$ is flag then $\operatorname{lk}_{K}(v)$ is an induced subcomplex of $K$ and it is also flag. The decomposition

\footnotetext{
${ }^{1}$ While this paper was under review the author came up with a computer-aided proof that in fact $m_{p}\left(\mathbf{P}^{2}\right)=13$. The details will appear in a future paper.
} 


$$
K=(K \backslash v) \underset{\operatorname{lk}_{K}(v)}{\bigcup} \mathrm{st}_{K}(v)
$$

together with the contractibility of $\operatorname{st}_{K}(v)$ give a Mayer-Vietoris sequence

$$
\cdots \rightarrow \widetilde{H}_{i+1}(K) \rightarrow \widetilde{H}_{i}\left(\operatorname{lk}_{K}(v)\right) \rightarrow \widetilde{H}_{i}(K \backslash v) \rightarrow \widetilde{H}_{i}(K) \rightarrow \widetilde{H}_{i-1}\left(\operatorname{lk}_{K}(v)\right) \rightarrow \cdots
$$

The following lemma is a reformulation of [6, Lem.5.3].

Lemma 2.1 Suppose that $K$ is a flag simplicial complex.

(a) If $K$ has $n \geq 0$ vertices then $\widetilde{H}_{i}(K ; \Lambda)=0$ for all $i>\frac{n}{2}-1$ and all groups $\Lambda$. (b) If $K$ has $2 n+2$ vertices and $\widetilde{H}_{n}(K ; \Lambda) \neq 0$ for some group $\Lambda$ then $K=O_{n+1}$.

Proof By a direct verification part (a) holds for $n=0$. Now suppose $K$ is a flag complex with $n$ vertices. If $K=\Delta_{n-1}$ then the conclusion of (a) holds. Otherwise there is a vertex $v$ which is not adjacent to at least one other vertex, so that $\# V\left(\operatorname{lk}_{K}(v)\right) \leq n-2$. We have $\# V(K \backslash v)=n-1$, so by induction $\widetilde{H}_{j}(K \backslash v ; \Lambda)=0$ for $j>\frac{n}{2}-\frac{3}{2}$ and $\widetilde{H}_{j}\left(\mathrm{k}_{K}(v) ; \Lambda\right)=0$ for $j>\frac{n}{2}-2$. Together with the exact sequence (5) this implies (a).

Now let $K$ be as in part (b). The result holds when $n=0$ since $S^{0}=O_{1}$ is the only flag complex with 2 vertices and nontrivial $\widetilde{H}_{0}(K)$. Suppose $n \geq 1$ and again choose $v$ to be a vertex with \#V( $\left.\operatorname{lk}_{K}(v)\right) \leq 2 n$. Since $K \backslash v$ has $2 n+1$ vertices part (a) implies that $\widetilde{H}_{n}(K \backslash v ; \Lambda)=0$. Using (5) we get $\widetilde{H}_{n-1}\left(\mathrm{lk}_{K}(v) ; \Lambda\right) \neq 0$. By (a) the complex $\operatorname{lk}_{K}(v)$ must have exactly $2 n$ vertices and an inductive application of (b) yields $\mathrm{k}_{K}(v)=O_{n}$. If $w$ is the unique vertex of $K$ non-adjacent to $v$ then repeating the same argument shows that $w$ and $v$ have the same link and therefore $K=\Sigma O_{n}=O_{n+1}$. This completes the proof.

The non-flag counterpart of Lemma 2.1 is obvious for dimensional reasons and we leave it to the reader.

Lemma 2.2 Suppose that $K$ is any simplicial complex.

(a) If $K$ has $n \geq 1$ vertices then $\widetilde{H}_{i}(K ; \Lambda)=0$ for all $i>n-2$ and all groups $\Lambda$.

(b) If $K$ has $n+2$ vertices and $\widetilde{H}_{n}(K ; \Lambda) \neq 0$ for some group $\Lambda$ then $K=\partial \Delta_{n+1}$.

Proposition 1.1 now follows immediately from Lemmas 2.1, 2.2 and the inequality $m_{f}(X) \leq m_{p}(X)$.

Next we prove the stabilization result of Theorem 1.2. First observe that for any space $X$ we have the inequalities

$$
m_{s}(\Sigma X) \leq m_{s}(X)+1, \quad m_{f}(\Sigma X) \leq m_{f}(X)+2, \quad m_{p}(\Sigma X) \leq m_{p}(X)+2 .
$$

The second and third inequality are obvious since $\Sigma X$ is constructed by joining $X$ with $S^{0}$ or by adding two incomparable maximal elements to the representing poset. 
The first inequality holds since, for a simplicial complex $K$, the suspension $\Sigma K$ can be realized (up to homeomorphism) with just one extra vertex using the construction known as the one-point suspension [5]. Note that this construction does not preserve flagness.

There is no reason why the inequalities in (6) should always be equalities. Indeed, if $X=\bigvee^{n} S^{0}$ is the discrete space with $n+1$ points then $m_{s}(X)=m_{f}(X)=m_{p}(X)=$ $n+1$ but it is easy to see that $\Sigma X \simeq \bigvee^{n} S^{1}$ can be realized simplicially with asymptotically $\sqrt{2 n}$ (or $2 \sqrt{n}$ for a flag complex) vertices. The exact value of $m_{p}(X)$ in this case is worked out in [3, Rmrk.4.8]. However, the inequalities (6) always turn into equalities stably.

Proposition 2.3 For any non-acyclic space X of finite type there is a $k_{0}=k_{0}(X)$ such that for all $k \geq k_{0}$ we have

$$
\begin{aligned}
& m_{s}\left(\Sigma^{k} X\right)=m_{s}\left(\Sigma^{k-1} X\right)+1, \\
& m_{f}\left(\Sigma^{k} X\right)=m_{f}\left(\Sigma^{k-1} X\right)+2, \\
& m_{p}\left(\Sigma^{k} X\right)=m_{p}\left(\Sigma^{k-1} X\right)+2 .
\end{aligned}
$$

Proof We prove the result for $m_{f}(X)$. The remaining proofs are similar. Consider the sequence

$$
c_{f}(X), c_{f}(\Sigma X), c_{f}\left(\Sigma^{2} X\right), c_{f}\left(\Sigma^{3} X\right), \ldots
$$

By Proposition 1.1 this is a sequence of non-negative integers. Moreover, it is nonincreasing, since

$$
\begin{aligned}
c_{f}\left(\Sigma^{k} X\right)-c_{f}\left(\Sigma^{k-1} X\right) & =m_{f}\left(\Sigma^{k} X\right)-m_{f}\left(\Sigma^{k-1} X\right)-2\left(h\left(\Sigma^{k} X\right)-h\left(\Sigma^{k-1} X\right)\right) \\
& =m_{f}\left(\Sigma^{k} X\right)-m_{f}\left(\Sigma^{k-1} X\right)-2 \leq 0 .
\end{aligned}
$$

We used the fact that $h\left(\Sigma^{k} X\right)=h\left(\Sigma^{k-1} X\right)+1$ for every $k$ and every non-acyclic space $X$ and the inequality of (6). Hence the sequence must stabilize, so for sufficiently large $k$ we have $c_{f}\left(\Sigma^{k} X\right)=c_{f}\left(\Sigma^{k-1} X\right)$ and by the same calculation it implies $m_{f}\left(\Sigma^{k} X\right)=m_{f}\left(\Sigma^{k-1} X\right)+2$.

Theorem 1.2 is an immediate corollary of the last proposition.

We proceed with the proof of Proposition 1.3.

Proof of Proposition 1.3 The proof is constructive. Let $X=\Delta_{2 n}^{(n-1)}$ be the $(n-1)$ dimensional skeleton of $\Delta_{2 n}$. It is well known to be homotopy equivalent to the wedge sum of $\left(\begin{array}{c}2 n \\ n\end{array}\right)$ copies of the sphere $S^{n-1}$. By construction $m_{S}(X) \leq 2 n+1$.

Let $K$ be the smallest flag complex homotopy equivalent to $X$ and denote $m=$ $\# V(K)$. It is an easy consequence of the results of [8, Thm. 3.4] (see also [1] for a short proof) that a flag complex $K$ with $m$ vertices satisfies

$$
\operatorname{dim} \widetilde{H}_{*}(K ; \mathbb{Q}) \leq 2^{2 m / 5} .
$$


Using standard lower bounds for the binomial coefficients we get

$$
\frac{2^{2 n}}{\sqrt{4 n}} \leq\left(\begin{array}{c}
2 n \\
n
\end{array}\right)=\operatorname{dim} \widetilde{H}_{*}(X ; \mathbb{Q})=\operatorname{dim} \widetilde{H}_{*}(K ; \mathbb{Q}) \leq 2^{2 m / 5}
$$

which implies $m \geq 5 n-\frac{5}{4} \log _{2} n-\frac{5}{2}$ and

$$
\frac{m_{f}(X)}{m_{s}(X)} \geq \frac{m}{2 n+1} \geq \frac{5}{2}-\epsilon
$$

for sufficiently large $n$, depending on $\epsilon$.

To prove Proposition 1.4 we need an analogue of the characterization in Lemma 2.1(b).

Lemma 2.4 Suppose $K$ is a flag simplicial complex with $2 n+3$ vertices and $\widetilde{H}_{n}(K ; \Lambda) \neq 0$ for some group $\Lambda$. Then either $K$ is of the form

$$
O_{n+1} \bigcup_{L} C L
$$

where $L \subsetneq O_{n+1}$ is a proper induced subcomplex, or $K$ is isomorphic to the complex

$$
C_{5} * \underbrace{S^{0} * S^{0} * \cdots * S^{0}}_{n-1} .
$$

Recall that $C_{5}$ is the simplicial 5-gon hence $C_{5} * S^{0} * \cdots * S^{0}$ is a single edge subdivision of $O_{n+1}=\left(S^{0} * S^{0}\right) * S^{0} * \cdots * S^{0}$ along an edge in the 4-cycle $S^{0} * S^{0}$. Note that the converse of the lemma also holds: each of the complexes $K$ as above has $2 n+3$ vertices and $\widetilde{H}_{n}(K ; \mathbb{Z})=\mathbb{Z}$.

Proof First suppose that there is a vertex $v$ in $K$ with $\widetilde{H}_{n-1}\left(\mathrm{lk}_{K}(v) ; \Lambda\right)=0$. By the long sequence (5) we get $\widetilde{H}_{n}(K \backslash v ; \Lambda) \neq 0$. But $K \backslash v$ has $2 n+2$ vertices, so Lemma 2.1(b) gives $K \backslash v=O_{n+1}$ and so $K$ is of the form (7) with $L=\mathrm{lk}_{K}(v)$. The subcomplex $L$ must be proper as otherwise $K=C O_{n+1}$ would be a cone, contradicting $\widetilde{H}_{n}(K ; \Lambda) \neq 0$.

It therefore remains to consider the case when for every vertex $v$ we have $\widetilde{H}_{n-1}\left(\mathrm{lk}_{K}(v) ; \Lambda\right) \neq 0$. By Proposition 1.1 we get that $\# V\left(\mathrm{lk}_{K}(v)\right) \geq 2 n$ for all $v$. Moreover we can assume that $\# V\left(\operatorname{lk}_{K}(v)\right) \leq 2 n+1$, as otherwise $K$ would be a cone, hence a contractible space, contradicting $\widetilde{H}_{n}(K ; \Lambda) \neq 0$. A parity check shows that there exists at least one vertex $v$ of degree exactly $2 n$, i.e. with $\# V\left(\operatorname{lk}_{K}(v)\right)=2 n$. By Lemma 2.1(b) there is an isomorphism $\mathrm{lk}_{K}(v)=O_{n}$. Let $u_{1}, \ldots, u_{2 n}$ be the vertices of $\mathrm{lk}_{K}(v)$ and let $x, y$ be the remaining two vertices of $K$ non-adjacent to $v$.

Let $G=\overline{K^{(1)}}$ so that $K=\operatorname{Ind}(G)$. By our previous remarks $G$ has $2 n+3$ vertices $\left\{v, u_{1}, \ldots, u_{2 n}, x, y\right\}$, each of degree 1 or 2 , such that $u_{1}, \ldots, u_{2 n}$ are non-adjacent to $v$, the subgraph induced by $\left\{u_{1}, \ldots, u_{2 n}\right\}$ consists of $n$ disjoint edges and $v$ is adjacent to $x$ and $y$. Up to isomorphism there are only four such graphs, shown in Fig. 2, each formed by a number of disjoint edges and one bigger component which is $P_{3}, P_{5}, P_{7}$ 
Fig. 2 Complements of the 1 -skeleton of $K$ in the proof of Lemma $2.4(n=4)$

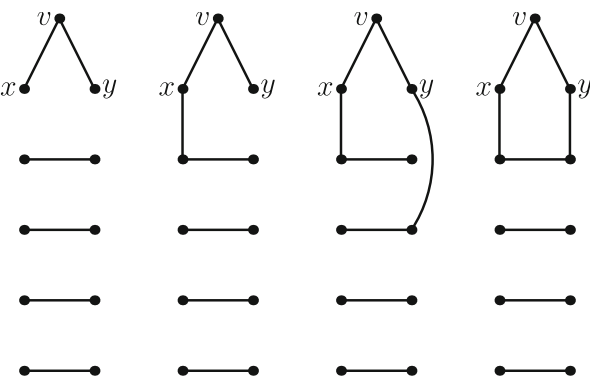

or $C_{5}$. An easy case analysis shows that in the first three cases the graph $G \backslash N[x]$ has an isolated vertex, so that $\operatorname{lk}_{K}(x)=\operatorname{Ind}(G \backslash N[x])$ is a cone, contradicting our assumption about non-triviality of $\widetilde{H}_{n-1}$ of all links. Since the complement of $C_{5}$ is again a $C_{5}$, that proves that in the fourth case $K$ has the form (7).

We are now ready to prove Proposition 1.4.

Proof of Proposition 1.4 To prove (a) note that $L \subseteq \partial \Delta_{n+1}$ and that the mapping cone $K=\partial \Delta_{n+1} \cup_{L} C L$ of the inclusion $L \hookrightarrow \partial \Delta_{n+1}$ can be realized using $n+3$ vertices. If $L$ is a proper subcomplex then $L \hookrightarrow \partial \Delta_{n+1}$ is null-homotopic and therefore $K \simeq \partial \Delta_{n+1} \vee \Sigma L$. Since $\Sigma L$ is non-acyclic we get $K \not S^{n}$ and $c_{S}(K) \neq 0$ by Proposition 1.1. At the same time $c_{s}(K) \leq(n+3)-n-2=1$, so we conclude $c_{S}(K)=1$. If, on the other hand, $L$ is not proper, that is $L=\partial \Delta_{n+1}$, then $S^{n} \vee \Sigma L=S^{n} \vee S^{n+1}$ and the result follows from the previous argument by inverting the roles of the two wedge summands.

Next we prove (b). If $c_{f}(X)=1$ then $X$ is homotopy equivalent to a flag complex $K$ which satisfies the assumptions of Lemma 2.4. If $K=C_{5} * S^{0} * \cdots * S^{0}$ then $K$ is homeomorphic to a sphere and $c_{f}(K)=0$, therefore we must have $K=O_{n+1} \cup_{L} C L$ for a proper induced subcomplex $L \subsetneq O_{n+1}$. Note that $L$ is either contractible (if it contains exactly one vertex from some of the join factors $S^{0}$ ) or it is isomorphic to $O_{m}$ for $0 \leq m \leq n$.

If $L$ is contractible then attaching a cone over $L$ does not affect the homotopy type and then $K \simeq S^{n}$ with $c_{f}(K)=0$. Otherwise the inclusion $L=O_{m} \hookrightarrow O_{n+1}$ is null-homotopic and we have a homotopy equivalence

$$
K=O_{n+1} \bigcup_{O_{m}} C O_{m} \simeq O_{n+1} \vee \Sigma O_{m}=S^{n} \vee \Sigma S^{m-1}=S^{n} \vee S^{m}
$$

Finally when $c_{p}(X)=1$ then the inequality $c_{f}(X) \leq c_{p}(X)$ together with the last statement of Proposition 1.1 imply $c_{f}(X)=1$. The construction of models for $S^{n} \vee S^{m}$ follows from the argument and this completes the proof.

\section{References}

1. Adamaszek, M.: Maximal Betti Number of a Flag Simplicial Complex. arxiv/1109.4775

2. Barmak, J.A.: Algebraic Topology of Finite Topological Spaces and Applications, Lecture Notes in Mathematics, vol. 2032. Springer, Berlin-Heidelberg (2011) 
3. Barmak, J.A., Minian, E.G.: Minimal finite models. J. Homotopy Relat. Struct 2(1), 127-140 (2007)

4. Hardie, K.A., Vermeulen, J.J.C., Witbooi, P.J.: A nontrivial pairing of finite $T_{0}$ spaces. Topol. Appl. 125(3), 533-542 (2002)

5. Joswig, M., Lutz, F.H.: One-point suspensions and wreath products of polytopes and spheres. J. Combin. Theory Ser. A 110(2), 193-216 (2005)

6. Kahle, M.: Topology of random clique complexes. Discrete Math. 309(6), 1658-1671 (2009)

7. Katzman, M.: Characteristic-independence of Betti numbers of graph ideals. J. Combin. Theory Ser. A 113, 435-454 (2006)

8. Kozlov, D.: Convex hulls of $f$ - and $\beta$-vector. Discrete Comput. Geom. 18(4), 421-431 (1997)

9. Kozlov, D.: Combinatorial Algebraic Topology Algorithms and Computation in Mathematics, vol. 21. Springer, Berlin, Heidelberg (2008)

10. May, J.P.: Lecture notes about finite spaces for REU (2003). http://math.uchicago.edu/ may/finite. html

11. McDuff, D.: On the classifying spaces of discrete monoids. Topology 18(4), 313-320 (1979)

12. Stong, R.E.: Finite topological spaces. Trans. Am. Math. Soc 123, 325-340 (1966)

13. Weng, D.: On minimal finite models, a REU paper. http://www.math.uchicago.edu/ may/VIGRE/ VIGRE2010/REUPapers/Weng.pdf 